Collaborative decision-making in multi-buy food purchases

Craig G Anderson, University of Stirling

Louise A Reed, University of St Andrews

Accepted refereed manuscript of:

Anderson CG \& Reid LA (2019) Collaborative decision-making in multi-buy food purchases. Journal of Cleaner Production, 216, pp. 520-527.

DOI: https://doi.org/10.1016/j.jclepro.2018.12.128

(C) 2018, Elsevier. Licensed under the Creative Commons Attribution-NonCommercialNoDerivatives 4.0 International http://creativecommons.org/licenses/by-nc-nd/4.0/ 


\begin{abstract}
This research presents a quasi-experiment utilizing an original card-game to investigate aspects implicated in the creation of food waste in the UK, including over purchasing due to a lack of advanced planning as well as susceptibility to advertising multi-buy offers. A card game embedding a clear trade-off between the temptation of additional gain and the risk of waste was devised. Decisions to purchase different multi-buy offers were recorded across individual and group decision-making. The results indicate that collaborative purchase decision-making could have some impact on the way in which the risk of waste is dealt with, consistent with social signaling and collective action. Compared to individual decision-making, group decision-making saw a deferral in choices to purchase multi-buy offers with an associated risk of wastefulness wen only a moderate temptation was added. These differences were embedded in both planned strategies and revealed behavior. Food waste is linked to marketing practices as well as household routines and perceived levels of control, particularly amongst households with multiple young adults. Shopping is typically conducted as an individual, however collaborative shopping experiences may help with planning and control, particularly amongst a student population.
\end{abstract}

Key Words: Food waste; decision-making; consumer behavior; risk; collaboration; deliberation.

\title{
Research Highlights:
}

- Household routines, multi-buy offers, and planning control can create food waste

- Results from a card game experiment show deferral of waste in group decision-making

- Collaborative shopping could reduce waste particularly in student housing 
Word Count: 7,937

\section{INTRODUCTION}

Wastefulness can be seen as either spending more money on an item than is necessary or where the purchaser does not fully utilize the item that has been purchased. It is hypothesized that people may eschew a behavior that is in their own best interest to avoid the appearance of wastefulness (Arkes, 1996). Despite an apparent social desire to not signal wastefulness, indications suggest that between $15 \%$ and $30 \%$ of the all purchased food in Europe and USA is wasted (Quested, and Johnson, 2009). In UK households, somewhat more than half of the food waste post-purchase occurs because the food was not used in time as part of overpurchasing (Williams et al., 2012). In behavioral studies, several reasons for food wastage are given, e.g., 'lack of plan' or 'change of plans', 'buying too much' (WRAP, 2007) which occur across planning, shopping, storage, preparation and consumption phases.

Underlying these reasons, however, are a range of social, structural and political factors, all of which have been well explored in academic literature across a range of disciplines. For example, in theory of planned behavior (Laureti, and Benedetti, 2018), heuristics (Lazzarini et al., 2018; Setti et. al., 2018), materialism (Diaz-Ruiz et al, 2018), motivation (Hüttel et al., 2018), and household rituals (Revilla and Salet, 2018). Thus, avoidable household waste is largely due to a combination of organizational, habitual, and other skills-based constraints, as well as external factors such as advertising (Mallinson et al., 2016; Quested et al., 2013). Despite advancements in research, and an understanding of why food waste occurs, policies and practices are failing to prevent it from continuing. There is thus an opportunity to further explore what can be done. For example, it is suggested future studies should focus on consumers' motivations for and barriers to choosing sustainable foods in relation to these different interventions and explore how to overcome these wide-spread biases. (Lazzarini, et. al., 2018). In addition, future research on rebound effects, loss aversion, and distaste for wastefulness is suggested (Hüttel et al., 2018). 
Innovative methods may be helpful in furthering this agenda, contributing to better understanding the issue at hand. For instance, calls have been made to find ways to test new ideas and interventions that could reduce food waste in households (Hebrok, and Boks, 2017). Experimentation has been proposed as a key way in which governance drives sustainability transitions, notably by creating space for innovative solutions to emerge (Kivimaa et al., 2017). Consequently, in this paper we aim to embed and test some aspects of loss aversion, wastefulness, motivation, and planning into experimental analysis.

\section{LITERATURE REVIEW}

Research into the issue of consumer food purchasing has surrounded key issues such as consumer decision-making and control; food consumption as a form of self-presentation; and, the impact or costs of such consumption We now discuss each of these in turn, paying specific attention to the latter, which we deem important in the context of the risk society.

Recent research has demonstrated the divergent lines of motivation that sustainable and non-sustainable decision-making follow (Hüttel et al., 2018). These motivations suggest that sustainable decision-making focuses on the avoidance of negative outcomes and that they are also often linked to making sacrifices, These literatures also suggest that purchasing that is unsustainable is more likely to result from compulsive intuition, involving low levels of control (Lee and Ahn, 2016). Likewise, perceived levels of control over time has been linked to reduced preparation time and consequently, greater consumption of impulsive and convenience food (Jabs and Devine, 2006) which is largely unsustainable. The issue of control is therefore central to scholarly discussions of food purchasing and waste, and it has been recommended that interventions should focus on increasing consumers' perceived behavioral control over food waste (Visschers et al., 2016). By control, these literatures refer to the perception the consumer has of how easy or difficult the behavior is, in other words, how likely is it that they 
will waste food, or be able to be able to use up the food they've purchased. Much of the discussion around perceived control can be traced back to the Theory of Planned Behavior (TPB), a psychological model, which suggests that control is a combination of self-efficacy and controllability (Ajzen,1991). Since the TPB, there have been numerous other attempts to explain and model human behavior, and food purchasing specifically, and the important lesson from these literatures is the complexity behind consumer decision-making, and the emphasis on the individual consumer.

A study into Italian purchasing behavior toward organic food products hinted roles for attitudes, subjective norms, and perceived behavioral control as suggested in the classical TBP (Laureti, and Benedetti, 2018). Purchasing can be seen as the most critical choice of the consumers' food waste. Gaps between behavior and outcomes add to uncertainty in food decisions, leading to heuristics. While retail can affect consumer behaviors relevant to household food waste, related experiences of other individuals are not visible, leading to individual heuristics (Setti et. al., 2018). Consumers' food choices are often based on simple heuristics (Schulte-Mecklenbeck et al., 2013; Thøgersen et al., 2012). Interventions may therefore be better spent developing simple rules for sustainable purchasing behavior. It is suggested that studies should focus on consumers' motivations for and barriers to choosing sustainable foods in relation to these different interventions and explore how to overcome these wide-spread biases. (Lazzarini et al., 2018)

Consumers' purchasing discipline, waste prevention behaviors, and materialism values are useful predictors of food waste behavior (Diaz-Ruiz et al., 2018). Saving money and economic awareness seems to be an important feature when it comes to assessing the effectiveness of collaboration and food sharing practices over waste reduction. Although noncollaborative behaviors may hinder food sharing effectiveness (Morone et al., 2018). The role of shopping experiences can affect WTP for sustainable goods (Zhang et al., 2018). Different 
human values are attached to consumers purchase decisions versus non-purchasing choices. Economically sustainable and non-sustainable decision-making follow divergent lines of motivational reasoning. Economically sustainable decision-making focuses on the avoidance of various negative outcomes such as sacrifice. However, consumers associate decisions to purchase unneeded or unaffordable products with instant happiness and future well-being. Future research on, loss aversion, and distaste for wastefulness is recommended (Hüttel et al., 2018).

Calls exist for a stronger integration of different disciplinary perspectives to look at why purchasing intention interventions to reduce food waste are not often successful. Household practices and non-conscious influences can be better predictors. A social practice approach adds to the perspective on food waste generation, moving beyond individual psychological factors such as attitudes, behavior, and choice (Schanes et al., 2018). Pressing circumstances and priorities such as the household's economic situation, busy schedules and the number or age of children in a household overshadow the preventive role that food rituals might have in saving food. This can be explained by practices of cooking and shopping for food being normally performed by only one member of the household (Revilla and Salet, 2018). Existing efforts that teach households to better manage food at home and avoid overpurchasing could be complemented with regulation of retailers' marketing tactics, for example, ensuring the continued presence of small food retailers for high-levels of physical access to food retail (Lee, 2018). However, calls suggest that help in better shopping planning would reduce household food waste. This result, compared with demographics, shows that younger respondents, and in particular those living with four or more adults, tend to think that shopping planning would help to reduce their household food waste, including nudging proposals (Mallinson, et al., 2016; von Kamke and Fischer, 2018).

The emphasis on the individual consumer has been widely critiqued (Evans, 2011; 
Prothero et al., 2011) and countered by arguments for an environmental rationality that is common and shared rather than individualistic (Leff, 2012). Communities, social action as well as intra and inter personal debates are also important considerations as to why individuals cannot make consumption changes (Hobson, 2002). For instance, that retail stores offer large packages and "getting one for free" bargains, and food manufactures produce oversized ready to eat meals (Stuart, 2009), mean there are strong incentives to encourage shoppers to buy excess food by tempting them into believing they are getting a good deal (Burn, 2011). That these phenomena exist is a social and political matter, so a focus on changing individuals' and their food purchasing and waste activities is perhaps misguided. Indeed, those concerned with more sociological and political aspects of consumer practices identify other aspects of modern life which impact on consumer practices (Connolly and Prothero 2008). Amongst these are concepts such as risk (Halkier, 2001; Tulloch and Lupton, 2002), the collective and conventional nature of consumption (Warde, 2005), as well as the morality of consumption (Miller, 2001).

Commentary in research into cleaner production considers "why worry about some risks, which are not immediate and do not menace visibly?" (Danihelka, 2004, p583). One of the most influential considerations for the emergence of a radical ecologist perspective is based on the potentials of 'risk society' (Hannigan, 1995, p. 12). In this theory, individuals act in the face of risk and indeed act to avoid risk (Beck et al., 1994). It is therefore important to consider how individuals respond to potential risk as a consequence of their consumer behavior. It is important for proposed solutions such as a 'green consumerism' and choice architecture through 'nudging' (Thaler and Sunstein, 2008), or more radical solutions like a progressive consumption tax (Frank, 1999). In addition to the analysis of responses to risk, theoretical considerations of the collective and individual action also need to be considered. 


\section{AIMS}

There is a likelihood of heuristics and individual biases being used in food purchasing decisions (Lazzarini et al., 2018; Setti et al., 2018; Schulte-Mecklenbeck et al., 2013; Thøgersen, et al., 2012). Signaling waste, saving money, planning, and the individual nature of household food shopping, are important aspects in using collaboration to confront waste (Arkes, 1996; Morone et al., 2018; von Kamke and Fischer, 2018). Future research on loss aversion and distaste for wastefulness is recommended (Hüttel et al., 2018). The aim of this research was thus to investigate the ability to act in the face of a clear risk of waste being associated to purchasing in excess, in individual decision-making or as part of deliberation with others. These decisions to purchase in 'excess' would be taken in the context of multi-buy offer, where there is temptation and associated impulsion to purchase in excess.

Purchase behavior can be specific to nations (Foster et al., 2006; Ponis et al., 2017). It might be that consumerist patterns are specific to the UK, or that socio psychological factors pertaining to the social signaling of wastefulness may differ between countries. Thus, analysis aimed to investigate responses to a risk of waste being attached multi-buy offers given different prior exposure to shopping habits and beliefs formed around country of upbringing.

The question being investigated is whether forced deliberation through collaborative decision-making creates:

1. Different planned purchase strategies.

2. Changes in impulsivity towards multi-buy purchases given the trade-off between the temptation of extra gain and the risk of wastefulness. 


\section{METHODOLOGY}

\subsection{Quasi-Experimental Design}

The multi-faceted nature of consumption behaviours would have been more difficult to track by using composite scores on, beliefs, attitudes, income etc. via a survey, and would have required a much larger sample size. It was the intention to have participants play the game in person as an individual and collectively to investigate collaborative choices in the presence of other participants, making an online experiment less feasible. A quasi-experiment was therefore devised, using different compositions of groups of consumers from broadly the same national backgrounds. It was assumed that UK consumers would be embedded in similar consumption patterns. Those students who were classified as UK customers, were those who grew up in the UK, with formative years being seen as important in forming habits, attitudes and beliefs. Manipulating the proportion of those who had grown up in the UK was most practical given data collection at a UK university. A quasi-experiment was therefore devised utilizing a sample of 40 postgraduate students at University of St Andrews to test the research aims.

Experimental pilot studies have considered socio-psychological determinants of sustainable behaviors (Juárez-Nájera, et al., 2010). Sustainability experiments can be smallscale or larger-scale, with potential to contribute in theories of transition management or reflexive governance (Hildén et al., 2017; Weiland et al., 2017). Experimental designs are often used to test environmental behavior and decision-making (Brucks, and Van Lange, 2007; Corraliza and Berenguer, 2000; Ramsey, 1993). They are commonplace in behavioral research (Cooper, 1982; Smith, 1962), and importantly they are also common in consumer behavior research (Belk, 1975; Bellizzi et al., 1983; Spangenberg et al., 1996; Turley and Millman, 2000). 
Shopping behaviors tend to be specific to nations (Foster et al., 2008; Ponis et al., 2107), with shared exposure to retail trends, including habits and beliefs pertaining to multi-buy offers and waste. Formative years are important in forming habits and beliefs over the lifespan (Foster et al., 2003; Ozer and Gjerde, 1989) and attitudes to food waste can be influenced by behaviors in the parental home (Morone et al., 2018). Nationality, measured by country of upbringing, was thus used as a proxy for similar habits surrounding multi-buy purchases and waste. Deliberate manipulation of groups was used to test the effect of collective decision-making on individuals with similar prior exposure to food shopping habits and trends. In a true experiment, groups would be assigned at random. Different proportions of participants who grew up in the UK were assigned to groups, given the availability of UK raised students when collecting data at St Andrews University, UK. Multi-buy offers are implicated in food waste amongst UK consumers (Stuart, 2009). A student sample, likely to be living in shared accommodation amongst young adults, tends towards the presence of convenience consumers implicated in food-waste (Mallinson et al., 2016; von Kamke and Fischer, 2018).

The design aimed to test how multi-buy purchase decisions could be altered when the same participants made decisions as a group or as an individual. The groups were designed with different UK and non-UK weightings. Two groups of each weighting were created, with the individual and collective decision-making being reversed for each group with the same weighting. The results of the two groups with the same proportion of UK participants would cancel out any bias or learning created, by the reversed order effect in the other group of the same proportion. There were therefore two groups of all UK participants, two groups of three UK participants and one non-UK, two groups of two UK participants and two non-UK participants, two groups of one UK participant and three non-UK, as well as two groups of all non-UK participants. This totaled ten groups of four, $20 \mathrm{UK}$ and 20 non-UK participants. 
Groups with different UK weightings could be used to signal the effect of collaborative decision-making on individuals with different priors relating to waste and multi-buy purchases.

The quasi-experimental design of this experiment constrained the sample given specific nationality weightings and order of participation being reversed. A minimum of 80 participants would have been needed to increase the sample, presenting a challenge in acquiring the balance of nationalities. A postgraduate sample was most appropriate given their international nature. It was not possible to get results from the general population, both in terms of nationality weightings and the necessity to organize participation. The quasi-experimental design intended to test group decision-making. As group decision-making can be impacted by social interaction and conformity (Fisher, 1993; Janis, 1971), it was preferred to have participants partake in group decision-making and interact face to face. A large sampled, online, true experiment could track the effect of individual and group decision-making from range of prior exposure or beliefs about multi-buy offers and food waste. However, it would have obscured face to face interaction. A quasi-experimental design, using country of upbringing as a proxy for prior beliefs about multi-buy purchases in food shopping and waste was deemed most appropriate.

\subsection{Card Game Design}

Considering the social costs associated with signaling wastefulness (Arkes, 1996), an associated risk of making wasteful purchases was added to the experiment. The offsetting of negative outcomes such as signaling waste, and a need to make sacrifices in forgoing instant happiness in sustainable choices, (Hüttel et al., 2018) is consistent with tradeoffs between risks and temptation. Risk was therefore added in the face of the temptation of a multi-buy offer, representing a risk of waste. A set of cards was thus developed that represented different levels of 'multi-buy' temptation and associated risk. This was represented in the form of three card types. Card type one displayed a single green spot. Card type two presented one green spot and 
another green spot clearly labeled as free, with a clearly represented risk of a half red spot. Card type three showed one green spot, two green spots clearly labeled as free and again a half red spot representing risk. These three types of card are illustrated in figure 1.

FIGURE 1:

Cards Used in Experiment

Type 1 Cards

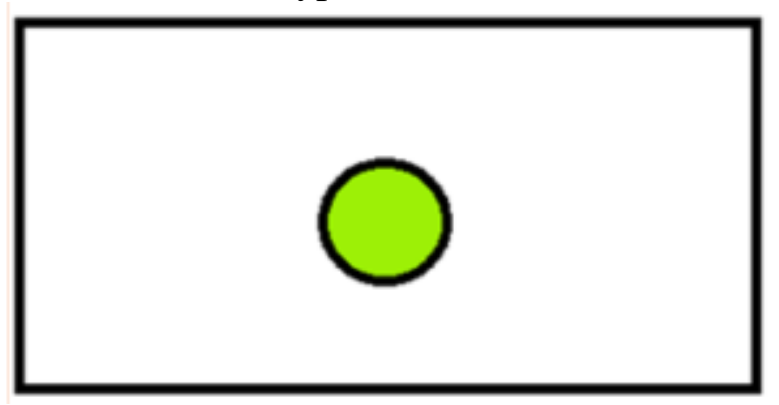

Type 2 Cards

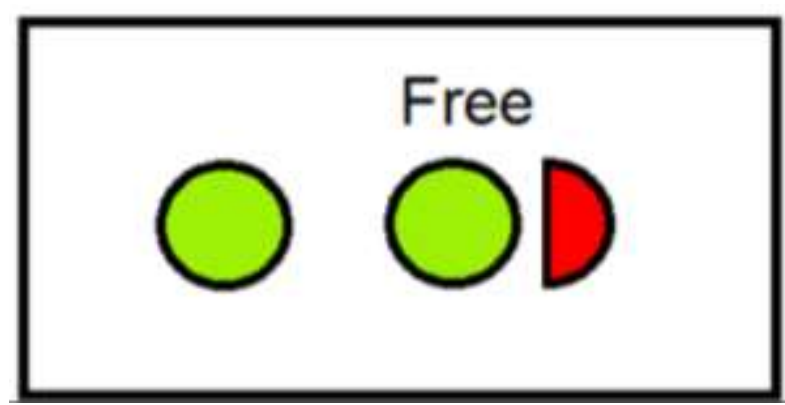

Type 3 Cards

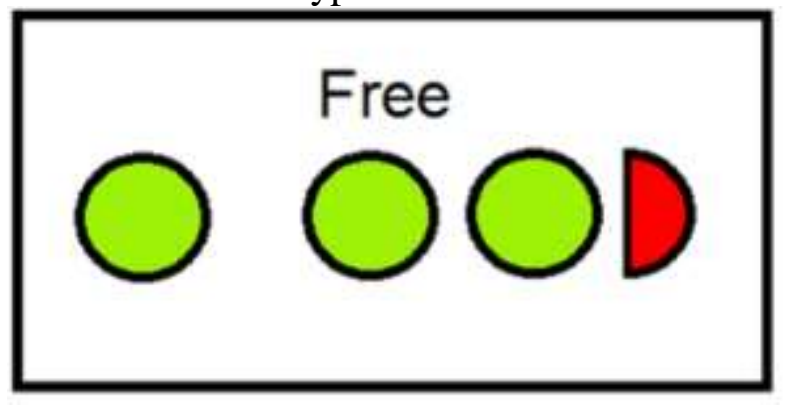

There was a total of 30 cards made, with twenty type one cards, eight type two cards and two type three cards. They were to be drawn in a set order every time, this allowed for equal analysis of strategy. When participants chose to purchase certain card types would have been obscured by having randomized sequences of cards. If high risk or multi-by cards appeared earlier or later in the deck from one participant to the next, the results would have been influenced by different learning and strategies created by these random changes. Keeping the appearance of multi-buy or higher risk cards consistent in their distribution across the sequence meant that strategies could be more fairly compared. The sequence of the cards was type $1,3,1,1,2,1,1,2,2,1,1,2,1,2,1,1,3,1,1,2,1,1,2,1,2,1,1,1,1,1$. 
Participants were told that they were given a starting purchasing power of 20 credits and that they would be offered to purchase a series of 30 cards. They were told that the aim of the game was to accumulate as many green spots as they could with their credit. They were also told that the purchasing of a card with a half red spot would see any future purchases thereafter increased by 0.5 credits, with the initial cost being 1 credit per card. Cards were drawn one by one and participants were asked whether they wished to purchase that card. They would be informed of how much credit they had remaining after each purchase. They were not given any information regarding potential strategies, and at the end of the game, they were asked what their strategy was, and their response was coded.

The most likely strategy for maximum score of twenty green spots was to avoid the costs associated with the multi-buy cards altogether, given the fixed sequence. Possible combinations did exist where multi-buy purchases could be purchased and the highest score could be equaled, but it could not be exceeded. Participants were not told that the maximum score was 20 as this could impact strategies upon the second play as either an individual or group depending on randomization. Participants were told there was a total of 30 cards. Concealment of the deck was used to hide the number of cards left in the deck, that could have impacted on strategies, as-per feedback in piloting the game. While it is probable that participants would have had a rough idea of how many cards were left, it was reported that visual confirmation of this led to changes in decision-making. If participants had been given an unknown number of cards rather than a known limit of 30, purchasing strategies might have naturally incorporated early attempts to learn the deck rather than reflecting a need to make a purchase decision. A known limit of 30 cards was intended to create a sense of urgency in accumulating credit, even at an early stage, avoiding the likelihood of many decisions to not purchase cards early on. 
It was also felt in piloting that being able to see the calculations of their credit score influenced their behavior, so a suitable recording sheet was devised to conceal calculations from participants. Respective losses and gains needed to trigger emotions of loss and gain in the purchaser, including risk. The spots to be positively accumulated were therefore colored green and the waste half spots were colored red, owing to expected associations of those colors with safe/risk or gain/loss (Elliot et al., 2007).

A short semi-structured interview was conducted post experiment to provide some feedback on the validity of the experimental design. It was important to ascertain whether the experiment has indeed reflected a sense of multi-buy purchase behavior and if the design had induced the sense of temptation and impulsivity in the face of balancing extra gain against risk. It was also useful to be able to gather information on any sense of wastefulness in buying the multi-buy cards. This information was also helpful in determining possible strategies taken by individuals and groups. Although it is noted that group responses might have been affected by group dynamics.

\section{RESULTS}

In processing the data for this study, a number of behaviors needed to be represented. This involved analysis of overall tendencies to purchase for the three types of card, as well as the planned and revealed strategies that might have existed. Again, the variables that needed to be considered were: risk of wastefulness, individual and collective purchase behavior, and UK decisions against non-UK decisions. In all cases the data was not always normally distributed, the two sets of responses were taken from the same population and not chosen at random. It was therefore necessary to use a non-parametric sign test to for significance in differences between independent variable groups. The decision to purchase cards was the dependent variable. 
In all analysis, the three types of card were considered as low temptation, no risk, medium temptation and risk, large temptation and risk. Card one, with one green spot, no additional free spot, and thus no cost of waste half red spot, was considered low temptation with no risk. The risk actually remains stable between card two and card three irrespective of additional gain amounts. The balance between potential losses and gains is relevant as per Prospect Theory (Kahneman and Tversky, 1979), resulting in potential losses being weighted more heavily than potential gains. However, it is the presence of risk that is being tested rather than any measure of risk aversion; this could not be tested in the game. It was the trade-off between the risk of waste that was being tested, given the temptation of additional gains. Card two with one green spot, an additional free spot and an associated risk of half a red spot, was considered to have a medium temptation and risk. Card three with one green spot, two additional free green spots and an associated risk of half a red spot, was considered large temptation and risk.

In table 1 , the percentage of cards purchased for each card type in individual and deliberated group participation are compared. The results here are intended indicate an overall tendency for purchasing cards given their relative temptation and risk. For example, a higher percentage of choices to purchase the medium temptation and risk cards could indicate a tendency to ignore the risk given a certain temptation, under either individual or collective group decisions. It could also be indicative of wider strategies, relative to the tendency to choose to purchase other card types, or not purchase at all. For every offer of a card in the deck, participants could choose to purchase or not purchase that card, building towards accumulating as many green spots with 20 credits across 30 cards. Once 20 credits were used up, the remaining cards in the deck were marked as out of credit. 
TABLE 1:

Mean $\%$ of Individual and Group Purchases

\begin{tabular}{lrrr}
\hline Card Type & Individual \% & Group \% & Sig. Individual, Group \\
\hline Low Temptation No & & & \\
Risk & & & \\
Yes, purchase the card & 42.50 & 51.50 & $0.017^{*}$ \\
No, do not purchase card & 20.50 & 37.50 & \\
Out of credit & 37.00 & 11.00 & \\
& & & 1.000 \\
High Temptation and & & & \\
Risk & & & \\
Yes, purchase the card & 75.00 & 70.00 & \\
No, do not purchase card & 20.00 & 30.00 & \\
Out of credit & 5.00 & 0.00 & \\
& & & \\
Medium Temptation and & & & \\
Risk & & & \\
Yes, purchase the card & 17.50 & 20.00 & \\
No, do not purchase card & 58.75 & 75.00 & \\
Out of credit & 23.75 & 5.00 & \\
\hline
\end{tabular}
$* p<0.05$

Table 1 shows the percentage of total purchases of card types within the game across individual and group purchasing decisions. The only significant result using a sign of purchase decisions for each card along the sequence of 30, was drawn from this analysis. There was a significant difference in the total purchases of low temptation, no risk cards between individual and group purchasing. However, this can be largely accounted for by both the higher acceptance and rejection of these cards in group decision-making. Medium temptation and risk cards were purchased later in the sequence in group decision-making, as per table 3 , resulting in a much lower out of credit score for low temptation and no risk cards at the end of the sequence. There was no significant difference between group and individual total purchases for the high temptation and medium temptation cards that contained the risk.

The overall accumulation in purchases of the different card types between individual and group decision-making, showed little difference between individual and group purchases. 
As indicated, the timing of when to purchase cards containing the risk of a penalty was important in overall accumulation of card purchases. The effect of collaborative deliberation was on when to purchase the medium temptation and risk cards.

TABLE 2:

Mean $\%$ of UK and Non-UK Purchases

\begin{tabular}{llll}
\hline Card Type & UK Mean \% & Non-UK \% & Sig. UK, Non-UK \\
\hline
\end{tabular}

\section{Low Temptation No \\ Risk}

Yes, purchase the card

No, do not purchase card

Out of credit

\section{High Temptation and}

\section{Risk}

Yes, purchase the card

No, do not purchase card

Out of credit

\section{Medium Temptation and \\ Risk}

Yes, purchase the card

No, do not purchase card

Out of credit

$\begin{array}{ll}43.50 & 42.00 \\ 18.00 & 30.00 \\ 38.50 & 28.00\end{array}$

0.126

$\begin{array}{lr}70.00 & 57.50 \\ 20.00 & 37.50 \\ 10.00 & 5.00\end{array}$

25.63

30.00

48.75

53.13

25.63
0.383

5.00

Table 2 shows the total purchases of each card type for UK and non-UK individuals. Again, it was the low temptation, no risk cards that showed the greatest difference, with much lower rejections of low temptation, no risk cards amongst UK individuals. However, this, and all other differences here, were not significant given the parameters of this study. Nevertheless, across all three card types, there was a higher tendency to not purchase across all three types of card amongst individuals not raised in the UK, resulting in lower out of credit scores. There is no indication here that this is relating to different responses to multi-buy offers or a potential 
risk of waste, as the same trend is in the low temptation, no risk cards. Non-UK participants were hesitant to purchase cards, although inference of impulsivity or cautiousness is difficult.

As aforementioned, whether participants chose to purchase cards with risk early was an important difference. Tables 3 and 4 show when in the sequence of 30 cards certain cart types were purchased with the highest frequency, for example at card 10 in the sequence.

TABLE 3:

Mode Card Chosen in Distribution across Sequence of Purchases for Individual and Group Decisions.

\begin{tabular}{|c|c|c|}
\hline Card Type & $\begin{array}{l}\text { Mode in Normal Distribution of } \\
\text { Individual Decisions (Card } \\
\text { Number in Sequence of } 30 \text { Cards) }\end{array}$ & $\begin{array}{l}\text { Mode in Normal Distribution of } \\
\text { Group Decisions (Card Number } \\
\text { in Sequence of } 30 \text { Cards) }\end{array}$ \\
\hline
\end{tabular}

\section{Low Temptation No Risk}

Yes, purchase the card

\section{High Temptation and}

\section{Risk}

Yes, purchase the card

\section{Medium Temptation and}

\section{Risk}

Yes, purchase the card

${ }^{a}$ Medium temptation and high risk cards were purchased later in group decision-making

Table 3 shows the point in the deck where choosing to purchase a card happened at the highest frequency along deck sequence for each card type given individual or group decisionmaking. As previously stated, group purchases tended to result in an earlier acceptance of low temptation, no risk cards, with a corresponding later acceptance of medium temptation and risk cards. Anecdotally, there is evidence to support that this was a planned strategy as highlighted 
in the post-experimental interviews. This was generally quoted as being to defer risk then try to maximize gain through taking risk towards the end of the game. However, risk remained constant between medium temptation and risk, and high temptation and risk cards, so these decisions and strategy were conditional on amounts of additional gain for taking that risk at different points in the game.

TABLE 4:

Mode Card Chosen in Distribution across Sequence of Purchases for UK and Non-UK Decisions.

\begin{tabular}{lll}
\hline & $\begin{array}{l}\text { Mode in Normal Distribution of } \\
\text { UK Decisions (Card Number in }\end{array}$ & $\begin{array}{l}\text { Mode in Normal Distribution of } \\
\text { Non-UK Decisions (Card Number } \\
\text { in Sequence of } 30 \text { Cards) }\end{array}$ \\
\hline Card Type & Sequence of 30 Cards) & Sequents \\
\hline
\end{tabular}

\section{Low Temptation No Risk}

Yes, purchase the card

\section{High Temptation and}

\section{Risk}

Yes, purchase the card

No, do not purchase card

\section{Medium Temptation and}

\section{Risk}

Yes, purchase the card

Table 4 shows the point in the sequence of 30 cards where there was the highest frequency of choosing to purchase for each card type for UK individual and non-UK individuals. There were no notable differences between these two independent variable groups.

At the end of participation, individuals and groups were asked to describe their purchasing strategies throughout the game. These strategies were coded into five different 
groups based on the descriptions that were given. These were treated as distinct categories in analysis, and there were no hierarchical or ordinal assumptions that some strategies should be given higher or lower scores.

TABLE 5:

Planned Strategies' Coded Risk Score

\begin{tabular}{lr}
\hline Independent Variables & Sig. Planned Strategies \\
\hline & \\
UK, Non-UK & 0.920 \\
Individual, Group & 0.344 \\
\hline
\end{tabular}

\section{Planned Strategy Coding:}

1. Avoid half red spots and risk

2. Defer risk or minimize risk

3. No strategy

4. Value for money

5. More for money, high number of green spots

Table 5 shows the difference between the coded risk scores of planned strategies. There was no significant difference between the planned strategies of UK and non-UK individuals, or individual and group strategies. based on coding self-reported strategies. However, the difference of planned strategies appears to broadly correlate with revealed strategies in tables 3 and 4 . There was a greater difference between individual and group participation, and no difference between participants who had been raised in the UK and those raised outside the UK.

\subsection{Post-Experimental Interviews}

The post-experimental interviews were intended to substantiate the validity of the experimental and innovative nature of the card game design. 
More than half of respondents expressed some level of connection between real world purchasing and the experimental card game when prompted. The most common connection was made between playing the game and buying multi-buy purchases, with that link sometimes being made to supermarket multi-buys. Some participants linked the study to making tradeoffs based on price and quality, weighing up pros, cons and moral implications, as well as a temptation and gathering instinct. The most precise association was expressed by participant number 2, a participant from the UK, who stated that "playing the game was like buying fruit on multi-buys, which is often promoted as such and then it can go to waste". Participant number 13, a participant from outside the UK, stated that "people often try to get value for money and then end up creating waste". A small proportion made no link between playing the game and real-world decision-making, with some expressing that they simply played the game.

Participants who were asked to reflect on their associations attached to the half red spots displayed on the game cards consistently attached a negative association. A number of responses considered the implications of the half red spots as a cost, premium, or tax. There was a proportion that associated the half red spots with waste, however this stood at less than twenty per cent of those who were asked. Participants also repeatedly expressed experiencing a sense of temptation when presented with the cards that had the additional free spots, despite acknowledging the risk associated.

There were also some less particular responses of interest. Participant 4 expressed that "a small negative aspect with a large return is an acceptable purchase". Significant to the design of the game, participant 11 clearly stated that their decisions were based on risk assessment throughout the game. 


\subsection{Summary of Results}

Despite restrictions placed sample size by the quasi-experimental design, and a need to use sign tests, potentially obscuring some analysis of statistical significance, some important indications were present in the data collected in the experiment.

The most notable of these is the deferral in purchasing the medium temptation and risk cards in deliberated group decision-making relative to the same individuals making purchase decisions on their own. The medium temptation and risk cards represented the most difficult trade-off as there was a smaller temptation relative to the risk. High temptation and risk cards presented as too good a deal, obscuring differences between individual and deliberated group purchase decisions. This was an important aspect in designing two kinds of card where risk remained stable but relative temptation changed. The difference was only picked up where the trade-off was more difficult. The deferral in purchasing medium temptation and risk cards was reflected in the planned strategies of participants', with group decision-making tending more towards an avoidance or deferral of taking risk. Although this was at times associated with risk-seeking based behavior towards the end of the sequence of cards in the game.

There were no significant differences found between those raised in the UK and those raised outside the UK. Strategies to purchase all types of card were similar. This similarity is especially clear in table 4 , where each card type was purchased with the highest frequency at very similar points in the card sequence. Nevertheless, participants from outside the UK decided not to purchase all three types of card more often. As this hesitance was present across all three types of card, including the low temptation no risk card, it is difficult to infer links to prior beliefs and exposure to multi-buy offers and waste.

In the qualitative feedback on the experiment, there were indications that the quasiexperimental design reflected, and prompted, some elements of purchasing of multi-buy offers on food, including the temptation of extra gain and the risk of waste. 


\section{DISCUSSION}

\subsection{Implications for Theory}

Unsustainable purchases are can result from compulsive intuition, and low levels of control (Hüttel et al., 2018; Lee and Ahn, 2016). Perceived levels of control are linked to unsustainable food practices (Jabs and Devine, 2006), with recommendations to increase consumers' behavioral control over food waste (Visschers et al., 2016). Pressing circumstances such as the household's economic situation and the age of household residents determine food waste rituals, often explained by cooking and shopping for food being performed by only one member (Revilla and Salet, 2018). In particular, households with more young adults think that shopping planning would help to reduce their household food waste, including nudging proposals (Mallinson et al., 2016; von Kamke and Fischer, 2018).

Related experiences of other individuals are not visible, leading to individual heuristics (Schulte-Mecklenbeck et al., 2013; Setti et. al., 2018; Thøgersen et al., 2012). Simple rules are recommended to overcome these biases. (Lazzarini et al., 2018). Economic awareness is an important feature of collaboration in food waste reduction (Morone et al., 2018). Also, social signaling of negative outcomes such as waste is important in using collective action to induce environmental behaviors (Arkes, 1996; Connolly and Prothero, 2008; Grauel, 2016; Halkier, 2001; Tulloch and Lupton, 2002; Warde, 2005).

The results here indicate that when confronted with multi-buy purchases and the associated risk of wastefulness, economic caution is exercised in collaborative decisionmaking. Trade-offs involving moderate temptation or gains, when the risk of waste is made prominent, are deferred in collaborative decision-making. These results were found amongst a student sample, likely to be living in shared accommodation with multiple young adults, tending towards the presence of convenience consumers implicated in food-waste (Mallinson et al., 2016; von Kamke and Fischer, 2018). 


\subsection{Implications for Policy and Practice}

Collaborative decision-making in shopping may therefore mediate some wastefulness when faced with temptation of multi-buy offers. Considering the 'sharing economy' and initiatives such as mealsharing.com and shareyourmeal.net, the strength of these platforms in reducing food waste might be to target those consumers less inclined to spend time on meal preparation and planning. Targeting these consumers is not typically an intention of these platforms. Indeed sharemeals.org, targeted at ensuring provision for college students, might reflect the gap in enabling certain consumers in their food preparation and planning, particularly amongst those living as houses of multiple young adults. An extension to this process, might be to have shared shopping experiences as part of the meal sharing experience, to give a greater control over the ability to plan meals and avoid waste. Promoting these platforms and practices as part of student life could be especially productive.

The results did not find any meaningful differences in relation to the temptation and risk of waste presented with multi-buy offers between participants raised in the UK and those raised elsewhere. Nevertheless, the results of study contained a high proportion of UK raised participants, including in the differences between individual and group decision-making. A lack of advanced planning as well as susceptibility to advertising including multi-buy offers are implicated in the creation of food waste in UK households (Mallinson et al., 2016; Quested et al., 2013, Stuart, 2009; WRAP 2007; 2009). Efforts to improve food waste in the UK could encourage better advanced planning through collaborative deliberation, and increasing control over impulsivity through social signaling of wastefulness as part of collaboration.

Individualistic shopping would need to be complemented with regulation of retailers' marketing tactics (Mallinson at al., 2016; Quested et al., 2013; Stuart, 2009). Additionally, examples from Asia suggest that presence of small food retailers and different levels of physical access to food retail have an impact on household habits (Lee, 2018). 


\section{REFERENCES}

Ajzen, I. (1991). The theory of planned behavior. Organizational Behavior and Human Decision Processes, 50(2), 179-211.

Arkes, H. R. (1996). The psychology of waste. Journal of Behavioral Decision Making, 9(3), 213-224.

Beck, U., Giddens, A., and Lash, S. (1994). Reflexive Modernisation: Politics, Tradition and Aesthetics in the Modern Social Order. California. Stanford University Press.

Belk, R. W. (1975). Situational variables and consumer behavior. Journal of Consumer Research, 2(3), 157-164.

Bellizzi, J. A., Crowley, A. E., and Hasty, R. W. (1983). The effects of color in store design. Journal of Retailing, 59(1), 21-45.

Brucks, W.M. and Van Lange, P.A.M. (2007). When prosocials act like proselfs in a commons dilemma. Personality and Social Psychology Bulletin, 33, 750-758.

Burn, G. (2011). Motivation for dummies. For Dummies.

Connolly, J. and Prothero, A. (2008). Green consumption life-politics, risk and contradictions. Journal of Consumer Culture, 8(1), 117-145.

Cooper, J. O. (1982). Applied behavior analysis in education. Theory Into Practice, 21(2) 114-118.

Corraliza, J. A., and Berenguer, J. (2000). Environmental values, beliefs, and actions a situational approach. Environment and Behavior, 32(6), 832-848.

Danihelka, P. (2004). Subjective factors of Cleaner Production-parallel to risk perception?. Journal of Cleaner Production, 12(6), 581-584.

Diaz-Ruiz, R., Costa-Font, M., and Gil, J. M. (2018). Moving ahead from food-related behaviours: an alternative approach to understand household food waste generation. Journal of Cleaner Production, 172, 1140-1151. 
Elliot, A. J., Maier, M. A., Moller, A. C., Friedman, R., and Meinhardt, J. (2007). Color and psychological functioning: The effect of red on performance attainment. Journal of Experimental Psychology: General, 136(1), 154-168.

Evans, D. (2011). Blaming the consumer-once again: the social and material contexts of everyday food waste practices in some English households. Critical Public Health, 21(4), 429-440.

Fisher, R. J. (1993). Social desirability bias and the validity of indirect questioning. Journal of Consumer Research, 20(2), 303-315.

Foster, C., Green K., Bleda, M., Dewick, P., Evans, B., Flynn, A., and Mylan, J. (2006). Environmental Impacts of Food Production and Consumption: A report to the Department for Environment, Food and Rural Affairs. Manchester Business School. London, Department for Environment, Food and Rural Affairs.

Foster, J. D., Campbell, W. K., and Twenge, J. M. (2003). Individual differences in narcissism: Inflated self-views across the lifespan and around the world. Journal of Research in Personality, 37(6), 469-486.

Frank, R.H. (1999) Luxury Fever: Money and Happiness in an Era of Excess. Princeton, New Jersey. Princeton University Press.

Grauel, J. (2016). Being authentic or being responsible? Food consumption, morality and the presentation of self. Journal of Consumer Culture, 16(3), 852-869.

Halkier, B. (2001). Consuming ambivalences consumer handling of environmentally related risks in food. Journal of Consumer Culture, 1(2), 205-224.

Hannigan, J. (1995). Environmental Sociology: A Social Constructionist Perspective. London. Routledge.

Hebrok, M., and Boks, C. (2017). Household food waste: Drivers and potential intervention points for design-An extensive review. Journal of Cleaner Production, 151, 380-392. 
Hildén, M., Jordan, A., and Huitema, D. (2017). Special issue on experimentation for climate change solutions editorial: The search for climate change and sustainability solutionsThe promise and the pitfalls of experimentation. Journal of Cleaner Production, 169, $1-7$.

Hobson, K. (2002). Competing discourses of sustainable consumption: Does the 'rationalisation of lifestyles' make sense? Environmental Politics, 11(2), 95-120

Hüttel, A., Ziesemer, F., Peyer, M., and Balderjahn, I. (2018). To purchase or not? Why consumers make economically (non-) sustainable consumption choices. Journal of Cleaner Production, 174, 827-836.

Jabs, J., and Devine, C. M. (2006). Time scarcity and food choices: an overview. Appetite, 47(2), 196-204.

Janis, I. L. (1971). Groupthink. Psychology Today, 5(6), 43-46.

Juárez-Nájera, M., Rivera-Martínez, J. G., and Hafkamp, W. A. (2010). An explorative sociopsychological model for determining sustainable behavior: Pilot study in German and Mexican Universities. Journal of Cleaner Production, 18(7), 686-694.

Kahneman, D. and Tversky, A. (1979). Prospect theory: An analysis of decision under risk. Econometrica: Journal of the Econometric Society, 47, 263-291.

Kivimaa, P., Hildén, M., Huitema, D., Jordan, A., and Newig, J. (2017). Experiments in climate governance-a systematic review of research on energy and built environment transitions. Journal of Cleaner Production, 169, 17-29.

Laureti, T., and Benedetti, I. (2018). Exploring pro-environmental food purchasing behaviour: An empirical analysis of Italian consumers. Journal of Cleaner Production, 172, 3367-3378. 
Lazzarini, G. A., Visschers, V. H., and Siegrist, M. (2018), How to improve consumers' environmental sustainability judgements of foods, Journal of Cleaner Production. DOI: $10.1016 /$ j.jclepro.2018.07.033

Lee, K. C. (2018). Grocery shopping, food waste, and the retail landscape of cities: The case of Seoul. Journal of Cleaner Production, 172, 325-334.

Lee, M. S., and Ahn, C. S. Y. (2016). Anti-consumption, materialism, and consumer wellbeing. Journal of Consumer Affairs, 50(1), 18-47.

Leff, E. (2012). Environmental rationality: Innovation in thinking for sustainability. In: Technological, Managerial and Organizational Core Competences: Dynamic Innovation and Sustainable Development. Nobre, S., Walker, D. and Harris, R. (eds.) Hershey, Pennsylvania. Business Science Reference.

Mallinson, L. J., Russell, J. M., and Barker, M. E. (2016). Attitudes and behaviour towards convenience food and food waste in the United Kingdom. Appetite, 103, 17-28.

Morone, P., Falcone P. M., Imbert, E., and Morone, A. (2018). Does food sharing lead to food waste reduction? An experimental analysis to assess challenges and opportunities of a new consumption model. Journal of Cleaner Production. 185, 749760.

Miller, D. (2001). The poverty of morality. Journal of Consumer Culture, 1(2), 225-243.

Ozer, D. J., and Gjerde, P. F. (1989). Patterns of personality consistency and change from childhood through adolescence. Journal of Personality, 57(2), 483-507.

Ponis, S. T., Papanikolaou, P. A., Katimertzoglou, P., Ntalla, A. C., and Xenos, K. I. (2017). Household food waste in Greece: A questionnaire survey. Journal of Cleaner Production, 149, 1268-1277. 
Prothero, A., Dobscha, S., Freund, J., Kilbourne, W. E., Luchs, M. G., Ozanne, L. K., and Thøgersen, J. (2011). Sustainable consumption: Opportunities for consumer research and public policy. Journal of Public Policy \& Marketing, 30(1), 31-38.

Quested, T. E., Marsh, E., Stunell, D., and Parry, A. D. (2013). Spaghetti soup: The complex world of food waste behaviours. Resources, Conservation and Recycling, 79, 43-51.

Quested, T., and Johnson, H. (2009). Household food and drink waste in the UK. Wastes \& Resources Action Programme (WRAP).

Ramsey, J. M. (1993). Students’ environmental behavior. Journal of Environmental Education, 24(3), 31-36.

Revilla, B. P., and Salet, W. (2018). The social meaning and function of household food rituals in preventing food waste. Journal of Cleaner Production. DOI: 10.1016/j.jclepro.2018.06.038

Schanes, K., Dobernig, K., and Gözet, B. (2018). Food waste matters-A systematic review of household food waste practices and their policy implications. Journal of Cleaner Production, 182, 978-991.

Schulte-Mecklenbeck, M., Sohn, M., de Bellis, E., Martin, N., and Hertwig, R. (2013). A lack of appetite for information and computation. Simple heuristics in food choice. Appetite, 71, 242-251.

Setti, M., Banchelli, F., Falasconi, L., Segrè, A., and Vittuari, M. (2018). Consumers' food cycle and household waste. When behaviors matter. Journal of Cleaner Production, $185,694-706$.

von Kameke, C., and Fischer, D. (2018). Preventing household food waste via nudging: An exploration of consumer perceptions. Journal of Cleaner Production, 184, 32-40

Smith, V. L. (1962). An experimental study of competitive market behavior. The Journal of Political Economy, 70(2), 111-137. 
Spangenberg, E. R., Crowley, A. E., and Henderson, P. W. (1996). Improving the store environment: do olfactory cues affect evaluations and behaviors? The Journal of Marketing, 60, 67-80.

Stuart, T. (2009). Waste - Uncovering the Global Food Scandal. London. Penguin Books.

Thaler, R. H. and Sunstein, C. R. (2009). Nudge: Improving Decisions About Health, Wealth, and Happiness. New York: Penguin Books

Thøgersen, J., Jørgensen, A. K., and Sandager, S. (2012). Consumer decision making regarding a "green" everyday product. Psychology \& Marketing, 29(4), 187-197.

Tulloch, J. and Lupton, D. (2002). Consuming risk, consuming science: The case of GM foods. Journal of Consumer Culture, 2(3), 363-383.

Turley, L. W. and Milliman, R. E. (2000). Atmospheric effects on shopping behavior: a review of the experimental evidence. Journal of Business Research, 49(2), 193-211.

Visschers, V. H., Wickli, N., and Siegrist, M. (2016). Sorting out food waste behaviour: A survey on the motivators and barriers of self-reported amounts of food waste in households. Journal of Environmental Psychology, 45, 66-78.

Warde, A. (2005). Consumption and theories of practice. Journal of Consumer Culture, 5(2), $131-153$.

Weiland, S., Bleicher, A., Polzin, C., Rauschmayer, F., and Rode, J. (2017). The nature of experiments for sustainability transformations: A search for common ground. Journal of Cleaner Production, 169, 30-38.

Williams, H., Wikström, F., Otterbring, T., Löfgren, M., and Gustafsson, A. (2012). Reasons for household food waste with special attention to packaging. Journal of Cleaner Production, 24, 141-148. 
WRAP. (2007) Food Behaviour Consumer Research: Quantitative Phase [online]. Available at: http://www.wrap.org.uk/content/food-behaviour-consumer-research-quantitativephase. Accessed: 23/02/18.

WRAP. (2009) Household Food and Drink Waste in the UK [online]. Available at: http://www.wrap.org.uk/node/13529. Accessed 23/02/18.

Zhang, B., Fu, Z., Huang, J., Wang, J., Xu, S., and Zhang, L, (2018) Consumers' perceptions, purchase intention, and willingness to pay a premium price for safe vegetables: A case study of Beijing, China, Journal of Cleaner Production. DOI: 10.1016 /j.jclepro.2018.06.273 